\title{
Estudo comparativo entre sutura mecânica e manual em brônquio após pneumonectomia esquerda em cães (Canis familiaris): uma avaliação anatomopatológica
}

Edson Azevedo SIMÕES ${ }^{1}$ Fabio Biscegli JATENE ${ }^{2}$ Angelo João STOPIGLIA ${ }^{1}$ Denise Tabacchi FANTONI ${ }^{3}$ José Luiz GUERRA ${ }^{4}$ Vera Demarchi AIELLO 5 Rosangela MONTEIRO ${ }^{3}$ Eduardo Toshio IRINO ${ }^{1}$ Denise Aya OTSUKI ${ }^{3}$ Danielle Harumy BINOKI ${ }^{1}$ Rodrigo Ramos de FREITAS ${ }^{1}$ Silvana Maria UNRUH ${ }^{6}$

\section{Correspondência para:}

EDSON AZEVEDO SIMÕES

Laboratório de Cirurgia Cardiotorácica

- LCCT

Departamento de Cirurgia

Faculdade de Medicina Veterinária e

Zootecnia da USP

Avenida Prof. Orlando Marques de Paiva, 87

Cidade Universitária Armando Salles de Oliveira

05508-270 - São Paulo - SP

edas@uol.com.br

Recebido para publicação: 14/11/2003 Aprovado para publicação: 23/08/2005

\author{
1 - Laboratório de Cirurgia Cardiotorácica - LCCT - Departamento de \\ Cirurgia da Faculdade de Medicina Veterinária e Zootecnia da \\ Universidade de São Paulo - SP \\ 2 - Departamento de Cardiopneumologia da Faculdade de Medicina da \\ Universidade de São Paulo - SP \\ 3 - Departamento de Cirurgia da Faculdade de Medicina Veterinária e \\ Zootecnia da Universidade de São Paulo - SP \\ 4 - Departamento de Patologia da Faculdade de Medicina Veterinária e \\ Zootecnia da Universidade de São Paulo - SP \\ 5 - Laboratório de Anatomia Patológica do Instituto do Coração - Hospital \\ das Clínicas - Faculdade de Medicina da Universidade de São Paulo - SP \\ 6 - Serviço de Radiologia do Hospital Veterinário da Faculdade de Medicina \\ Veterinária e Zootecnia da Universidade de São Paulo - SP
}

\section{Resumo}

O objetivo deste estudo foi realizar um estudo experimental comparando-se sob o ponto de vista anatomopatológico as suturas manual e mecânica em brônquio principal após pneumonectomia esquerda em cães. Foram utilizados 18 cães, sadios, machos e fêmeas, adultos, sem raça definida, pesando entre 9 e $27,5 \mathrm{~kg}$. Foram separados em 2 grupos de 9 cães, de acordo com o tipo de sutura empregada: Grupo A - sutura manual com fio polipropileno 5-0; Grupo B sutura mecânica com grampeador mecânico modelo TL-30. Cada grupo foi subdividido em 3 subgrupos de 3 animais, sendo estabelecido estudo temporal aos 7, 15 e 36 dias de pós-operatório, onde foi realizada avaliação anatomopatológica da cicatrização das suturas manual e mecânica. $\mathrm{Na}$ avaliação histopatológica foram avaliados, qualitativamente e semi-quantitativamente, intensidade da inflamação, fibrose, vasos neoformados e presença ou não de tecido de granulação, granuloma tipo corpo estranho e necrose. Os resultados encontrados foram analisados estatisticamente. Com relação à análise histopatológica, ocorreu a formação de granuloma tipo corpo estranho no coto brônquico esquerdo em $88,9 \%$ dos cães submetidos à sutura manual e em nenhum dos cães submetidos à sutura mecânica. Houve, ainda, diferença estatística significativa nos cães dos Grupos A e B em relação à intensidade da inflamação, sendo de maior intensidade nos cães submetidos à sutura manual. Concluiu-se que os 2 tipos de sutura promoveram cicatrização adequada do coto brônquico principal esquerdo, embora tenha ocorrido maior intensidade de inflamação e maior ocorrência de granuloma tipo corpo estranho nos cães submetidos à sutura manual.

\section{Introdução}

O desenvolvimento dos grampeadores
Palavras-chave: Sutura. Brônquios. Pneumonectomia. Cães. 
cirúrgicos de todo o mundo.

Em 1826, Denans ${ }^{1}$ utilizou anéis metálicos para efetuar uma anastomose intestinal, estudando esta técnica em cães.

Petz apud Schwartz ${ }^{2}$, Steichen e Ravitch $^{3}$, em 1924, desenvolveu o primeiro grampeador mecânico utilizado com êxito, pois promoveu impermeabilização e hemostasia adequadas, no entanto, causava a desvitalização pela tensão excessiva nos tecidos do estômago, duodeno e cólon.

Em 1960, o modelo americano TA30 foi desenvolvido, o qual é atualmente utilizado na confecção da sutura brônquica ${ }^{4}$.

Os grampeadores mecânicos modelo TA podem ser usados com sucesso nas ressecções pulmonares em cães e gatos ${ }^{5}$.

$\mathrm{Na}$ cirurgia pulmonar, no homem, os grampeadores mecânicos são utilizados nas ressecções pulmonares, lobectomias, pneumonectomias, biópsia, segmentectomias, ressecções em cunha e bulectomias ${ }^{6,7,8}$.

Em cães e gatos, as indicações da sutura mecânica na cirurgia pulmonar incluem neoplasia, consolidação do lobo pulmonar, abscessos, bronquiectasias, bulectomias, torção de lobo pulmonar, lesões traumáticas, lobectomia e pneumonectomia ${ }^{9}$.

Vários estudos foram realizados comparando sutura manual e mecânica.

Apesar de não ter feito um estudo comparativo entre sutura manual e mecânica, Amosov e Berezovski ${ }^{10}$, em 1961, realizaram ressecções pulmonares em humanos utilizando sutura mecânica, tendo uma incidência de 3,5\% de fístula brônquica.

Dart, Scott e Takaro ${ }^{11}$, em 1970, relataram uma incidência de $2,5 \%$ de fístulas broncopleurais na sutura mecânica e 7,5\% na sutura manual. Verificaram várias vantagens na utilização da sutura mecânica como redução do tempo cirúrgico, melhor controle da hemorragia e menor incidência de fístula brônquica.

Peterffy e Calabrese ${ }^{4}$, em 1979, compararam, em 2 grupos de pacientes humanos, a sutura manual e mecânica. A incidência de fístula brônquica no grupo de pacientes submetidos a sutura mecânica com o modelo TA-30 e do grupo submetido a sutura manual com categute foi de $1 \%$ e $3 \%$, respectivamente. Forrester-Wood ${ }^{12}$, em 1980, concluiu, através de seus estudos, que a utilização dos grampeadores mecânicos tem diminuído a incidência de fístula brônquica de $11 \%$ para 2,6\%. $\mathrm{Na}$ pneumonectomia direita houve uma redução de $16 \%$ para $5,2 \%$ e na pneumonectomia esquerda de $6 \%$ para $0,8 \%$ em pacientes humanos. Os grampeadores são fáceis de usar e o brônquio não precisa ser dissecado extensivamente, no entanto, há um risco de comprometimento da parede por tensão excessiva do brônquio.

Pêgo-Fernandes et al. ${ }^{13}$, em 1990, realizaram trabalho experimental em cães, comparando as suturas mecânica e manual em brônquios lobares. Não houve intercorrência intra ou pós-operatória. A presença de granuloma tipo corpo estranho apresentou uma incidência de 100\% no grupo suturado com polipropileno e $16 \%$ no grupo suturado mecanicamente.

Ferreira et al. ${ }^{14}$, em 1997, fizeram estudo experimental em cães comparando sutura convencional com fio de poliéster e sutura mecânica na lobectomia parcial pulmonar. Concluíram que a sutura mecânica diminui o tempo cirúrgico e permite melhor controle da aerostasia e hemorragia. Não observaram diferenças histopatológicas quanto à cicatrização.

Weissberg e Kaufman ${ }^{15}$, em 1992, relataram a superioridade e a rapidez na realização da sutura mecânica. Por outro lado, Hubaut et al. ${ }^{16}$, em 1999, realizaram revisão de 723 pacientes humanos submetidos a pneumonectomia para tratamento de neoplasia pulmonar. Concluíram que a sutura manual do coto brônquico é a técnica de eleição, apesar da sutura mecânica ser simples e rápida.

Asamura, Kondo e Tsuchiya ${ }^{17}$, em 2000, realizaram estudo comparativo entre sutura manual e mecânica, bem como, sutura mecânica comparando diferentes tipos de grampeadores após pneumonectomia em pacientes humanos. Este estudo demonstrou 
que a incidência de fístula brônquica entre sutura manual e mecânica, bem como, estudo comparativo entre diversos tipos de sutura mecânica utilizando vários modelos de grampeadores não foi estatisticamente significante.

O presente estudo teve como objetivo comparar, sob o ponto de vista anatomopatológico, as alterações macroscópicas e microscópicas das suturas mecânica e manual em brônquio após pneumonectomia esquerda em cães.

\section{Materiais e Métodos}

Foram utilizados neste estudo 18 cães (Canis familiaris), machos e fêmeas, adultos, sem raça definida, pesando entre 9 e 27,5 $\mathrm{kg}$, provenientes do Canil Central da Faculdade de Medicina Veterinária e Zootecnia da Universidade de São Paulo (FMVZ-USP). Foram avaliados clinicamente e submetidos a exames complementares através de amostras de sangue coletadas para hemograma, função hepática e renal, para posteriormente serem incluídos no presente estudo. Esses exames complementares foram novamente realizados com 7 dias de pósoperatório em todos os cães dos Grupos A e B.

No período que correspondeu a 24 horas antes da cirurgia experimental, foi realizada antibioticoterapia profilática através da administração de benzilpenicilina benzatina (Benzetacil ${ }^{(\mathrm{R})}$ - Wyeth)na dose de $40.000 \mathrm{U} / \mathrm{kg}$ por via subcutânea.

Após jejum hídrico e alimentar de $6 \mathrm{e}$ 12 horas, respectivamente, os cães receberam como medicação pré-anestésica acepromazina 0,2\% $\left(\right.$ Acepran $^{(R)}$ - Univet $S$ / A) na dose de $0,1 \mathrm{mg} / \mathrm{kg}$, por via intramuscular, 10 minutos antes da indução. A indução anestésica foi realizada através da utilização de propofol (Diprivan ${ }^{(\mathrm{R})}$ Laboratórios Wellcome - Zeneca Ltda.) na dose de $5 \mathrm{mg} / \mathrm{kg}$ por via intravenosa. Em seguida, foi realizada intubação seletiva, manutenção anestésica com agente inalatório sevofluorano (Sevorane ${ }^{(R)}$ Abbott Laboratórios do Brasil
Ltda.) e bloqueio neuromuscular com administração de rocurônio (Esmeron ${ }^{(\mathrm{R})}$ Akzo Organon Teknika) na dose de 0,6 mg/ $\mathrm{kg}$ por via intravenosa.

A ventilação mecânica foi regulada de maneira a manter pressão inspiratória média de $20 \mathrm{~mm} \mathrm{Hg}$ na freqüência de 15-20 movimentos por minuto e com fração inspiratória de oxigênio de $100 \%$.

Os cães foram submetidos à toracotomia lateral esquerda na altura do $5^{\circ}$ espaço intercostal. As costelas foram separadas com afastador tipo Finochietto infantil (Quinelatto ${ }^{(\mathrm{R})}-$ Brasil).

Os pulmões foram afastados lateralmente e dorsalmente em relação à cavidade pleural, sendo identificadas as estruturas do hilo pulmonar.

Após ligadura dos elementos vasculares do hilo, o brônquio foi dissecado e uma pinça atraumática foi colocada proximalmente ao local da secção. A seguir, o brônquio principal esquerdo foi seccionado e o pulmão ressecado. $O$ coto proximal foi cirurgicamente tratado de forma diferente, de acordo com a utilização da sutura manual ou mecânica, para o seu fechamento. Assim foram divididos em 2 grupos de 9 cães:

Grupo A - sutura manual do coto brônquico esquerdo com pontos separados "em 8" utilizando fio de polipropileno 5-O (Prolene 5-O ${ }^{(\mathrm{R})}$ - Ethicon - Johnson \& Johnson) (Figura 1).

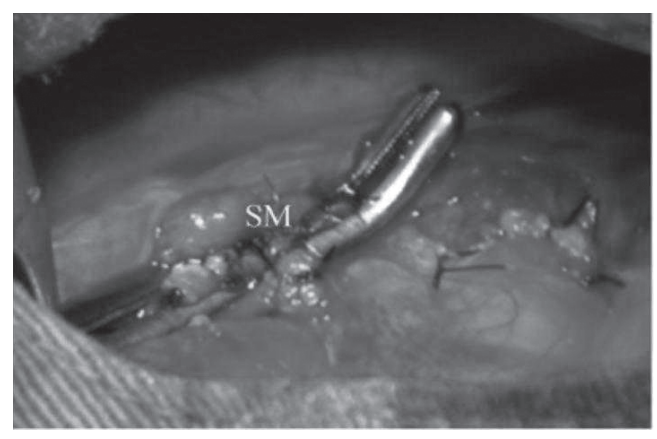

Figura 1 - Detalhe do brônquio principal esquerdo após pinçamento e sutura manual. Nota-se: SM: sutura manual

Grupo B - sutura mecânica do coto brônquico esquerdo com grampeador 
mecânico (Grampeador Mecânico TL-30 Ethicon - Johnson \& Johnson), grampos metálicos de $4 \mathrm{~mm}$, diầmetro do arame de $0,23 \mathrm{~mm}$, escala de intervalos de $2 \mathrm{~mm}$, com 11 grampos dispersos em fileira dupla (Figura 2).

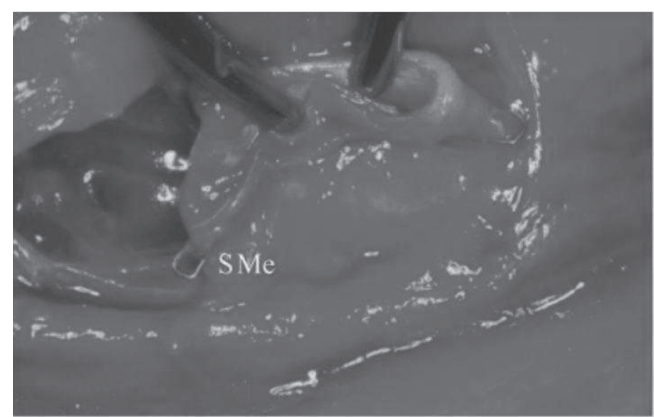

Figura 2 - Detalhe do brônquio principal esquerdo após sutura mecânica. Nota-se: SMe: sutura mecânica

Realizada a ressecção pulmonar (Figura 3), foi feita inspeção minuciosa do campo operatório para certificar-se da boa qualidade da hemostasia e aerostasia.

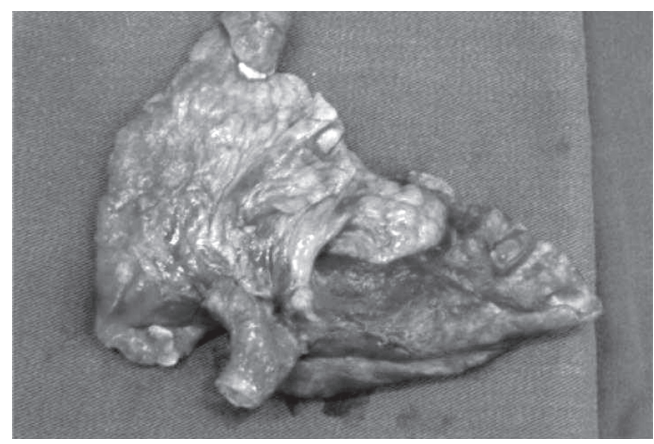

Figura 3 - Pulmão esquerdo ressecado após pneumonectomia esquerda em cão

Um dreno tubular sob selo d'água (Phisics ${ }^{(\mathrm{R})}-$ Brasil) foi colocado no espaço pleural.

A síntese da parede torácica foi realizada de acordo com técnica convencional. O dreno torácico foi mantido até que o animal estivesse estável e acordado.

Os cães foram encaminhados ao canil do Laboratório de Cirurgia Cardiotorácica da FMVZ-USP para cuidados pósoperatórios: analgesia, curativos da ferida cirúrgica e anotações dos parâmetros clínicos.
Foram mantidos com colar protetor até a retirada dos pontos.

Para avaliação anatomopatológica, cada grupo foi subdividido em 3 subgrupos de 3 animais, conforme o dia do sacrifício aos 7, 15 e 36 dias, respectivamente, sendo denominados de Subgrupo A (sutura manual com sacrifício aos 7 dias), Subgrupo A II (sutura manual com sacrifício aos 15 dias), Subgrupo $A_{\text {III }}$ (sutura manual com sacrifício aos 36 dias), Subgrupo $B_{\text {I }}$ (sutura mecânica com sacrifício aos 7 dias), Subgrupo $B_{\text {II }}$ (sutura mecânica com sacrifício aos 15 dias), Subgrupo $B_{\text {III }}$ (sutura mecânica com sacrifício aos 36 dias).

O aspecto macroscópico da sutura brônquica foi feito durante a necrópsia dos animais para coleta de material para avaliação histopatológica, onde foram avaliados graus de aderência, exsudato, deiscência, hemostasia, abscesso e fístula brônquica.

$\mathrm{Na}$ avaliação histopatológica, os segmentos de brônquios contendo as suturas foram retirados, identificados e preservados em solução de formol a $10 \%$.

Após secções transversais ao menor eixo do brônquio, os fragmentos obtidos foram submetidos ao processamento histológico convencional e submetidos à inclusão em parafina. Cortes de $5 \mathrm{~mm}$ de espessura foram, então, corados pela hematoxilina-eosina para estudo histológico.

As lâminas foram analisadas por um patologista, seguindo os padrões básicos de microscopia, observando-se intensidade da inflamação, fibrose, vasos neoformados e presença ou não de tecido de granulação, granuloma tipo corpo estranho e necrose mormente ao material de sutura utilizado. Os resultados para avaliação histopatológica obedeceram uma escala : + (leve); ++ (moderado); +++ (intenso). Cada variável foi estudada qualitativamente e semiquantitativamente.

\section{Resultados e Discussão}

As cirurgias foram realizadas conforme proposto no protocolo cirúrgico. 


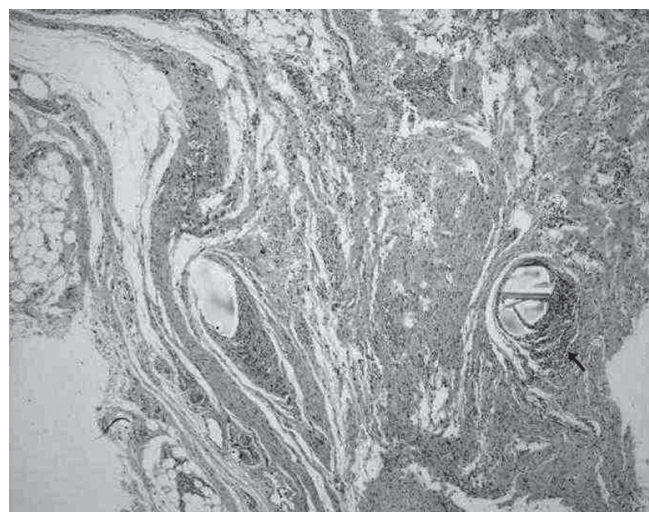

Figura 4 - Corte histológico de coto brônquico principal esquerdo após sutura manual. Observa-se moderado infiltrado inflamatórioao redor do fio de sutura Coloração: hematoxilina eosina (Objetiva: $5 x$ )

Ocorreu diferença estatística significativa entre os Grupos A e B, em relação à intensidade da inflamação e granuloma tipo corpo estranho (Figuras $4 \mathrm{e}$ $5)$.

A inflamação foi de maior intensidade nos cães submetidos à sutura manual.

Granuloma tipo corpo estranho ocorreu em $88,9 \%$ dos cães submetidos à sutura manual e em nenhum dos cães

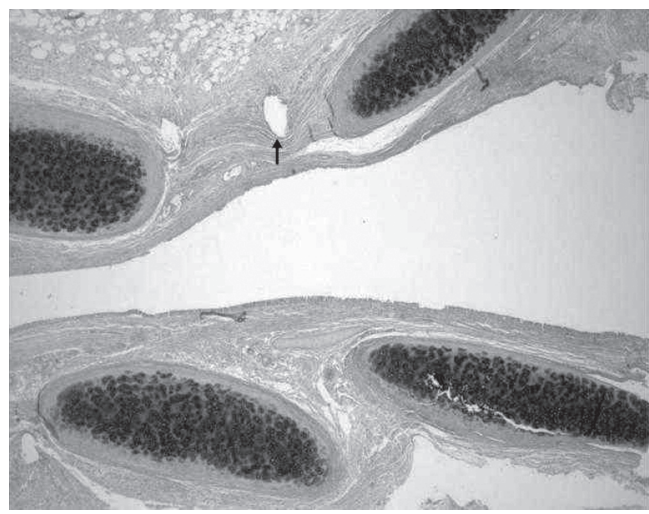

Figura 6 - Corte histológico de coto brônquico principal esquerdo após sutura mecânica. A seta mostra o espaço de onde foi retirado o grampo para processamento histológico. Quando comparado com a figura 4, o infiltrado inflamatório ao redor do espaço de onde foi retirado o grampo está praticamente ausente. Coloração hematoxilina eosina (Objetiva $5 \mathrm{x}$ )

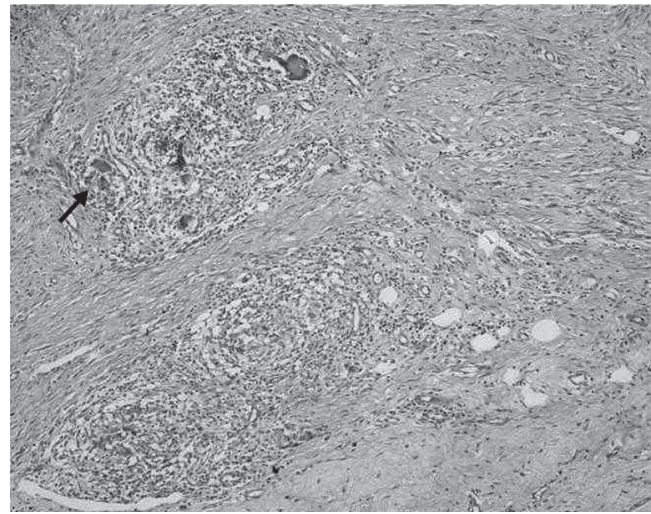

Figura 5 - Corte histológico de coto brônquico principal esquerdo após sutura manual. A seta indica a presença de granuloma tipo corpo estranho. Coloração: hematoxilina eosina (Objetiva 10x)

submetidos à sutura mecânica.

Em relação ao tecido de granulação, fibrose, necrose e neoformação vascular (Figuras 6 e 7) não houve diferença estatística significativa entre os 2 Grupos. O tecido de granulação estava presente nos tempos $7 \mathrm{e}$ 15 dias, desaparecendo nos cães sacrificados com 36 dias.

A avaliação macroscópica das feridas cirúrgicas das suturas da artéria pulmonar esquerda, brônquio, veias pulmonares e musculatura intercostal foi realizada conforme o dia da eutanásia aos 7, 15 e 36

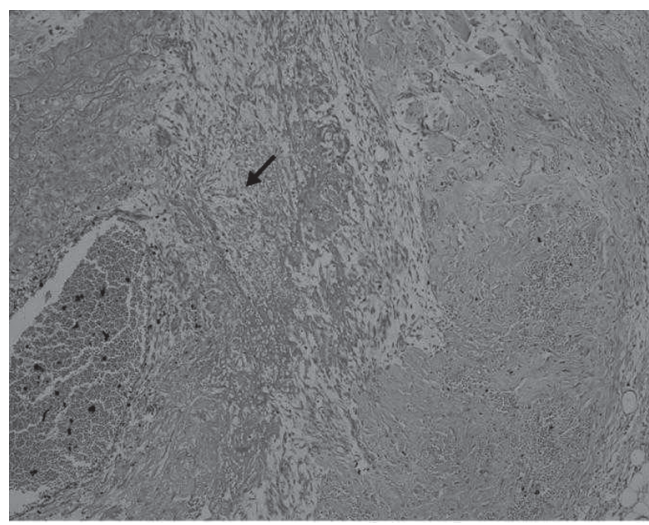

Figura 7 - Corte histológico de coto brônquico principal esquerdo após sutura manual. A seta mostra a presença de restos celulares necróticos. Coloração: hematoxilina eosinal' Objetiva 10x) 
dias de pós-operatório.

Todos os animais dos diferentes subgrupos não apresentaram deiscência da ferida operatória. Do mesmo modo, não se observou em nenhum animal sinais de infecção junto à sutura. tabela 1.

Os resultados estão representados na

A realização do presente estudo, permitindo a comparação entre a sutura mecânica e a sutura manual, está justificada diante do constante interesse médico e da escassez de trabalhos em Medicina Veterinária, contemplando as técnicas de sutura brônquica durante as ressecções pulmonares. Neste estudo experimental, realizado em cães hígidos, reduzimos o número de variáveis que pudessem favorecer uma cicatrização inadequada, contribuindo para a correta interpretação do processo de cicatrização que envolve a cirurgia pulmonar.

Nas intervenções cirúrgicas do brônquio, sejam por processos broncopulmonares neoplásicos, infecciosos ou traumáticos, a ocorrência de fístula brônquica é citada por vários autores $s^{3,4,12,16,17,18,19,20,21,22,23,24,25,26,27}$. A presença de deiscência e fístula brônquica não foi observada neste estudo.

Os materiais selecionados, fio de polipropileno para sutura manual e grampos de titânio para sutura mecânica, foram escolhidos, pois foram os mais utilizados na realização da sutura brônquica na literatura compulsada. Concordamos com Jatene et al. ${ }^{7}$, Peterffy e Calabrese ${ }^{4}$, Sawasaki et al. ${ }^{23}$ quando afirmam que o grau de irritação, capilaridade e tensão excessiva do material de sutura, assim como, a vascularização, irregularidade e infecção da linha de sutura são fatores importantes no aparecimento da fístula brônquica, obrigando a utilização de materiais e métodos que minimizem estas alterações.

A utilização de grampeadores mecânicos para a diminuição da incidência de fístula brônquica tem sido defendida por diversos autores. Como vantagem referem a necessidade de menor dissecção do brônquio, simetria dos grampos e da tensão no tecido bronquial, reação granulomatosa mínima, rapidez e linha de sutura radiograficamente visível ${ }^{4,5,7,10,12,14,26}$. Estas vantagens, em relação à utilização do grampeador mecânico, foram observadas neste estudo, principalmente no que se refere à simetria dos grampos, rapidez e linha de sutura radiograficamente visível. Além disso, a maior rapidez na confecção da sutura brônquica, através da utilização do grampeador mecânico, pode diminuir as complicações hemodinâmicas e anestésicas durante $\mathrm{o}$ ato operatório.

Embora a utilização da sutura mecânica seja segura, fácil e rápida, esta não está totalmente livre de complicações. A experiência do cirurgião e o posicionamento da carga do grampeador de maneira adequada, tornam-se importantes para evitar mal oclusão dos bordos da sutura e, consequentemente, escape de ar para o interior da cavidade torácica? ${ }^{9}$ No presente estudo, não se verificou as complicações acima mencionadas.

Resultados contraditórios foram apresentados por Hubaut et al. ${ }^{16}$. Concluíram que a sutura manual do brônquio após pneumonectomia é a técnica de eleição e, muitas vezes, até melhor que a sutura mecânica. De acordo com estes autores, a sutura manual com polipropileno é segura e seu custo é 10 vezes menor que a sutura mecânica, podendo ser facilmente adotada por todos os hospitais. Esta afirmação é válida, porque além de cumprir sua função em determinada cirurgia, o material de sutura deve ter um custo que facilite a realização deste procedimento cirúrgico em Medicina Veterinária.

No presente estudo, durante a necrópsia, realizada nos animais de ambos os grupos experimentais, estes não apresentaram deiscência ou fístula brônquica após pneumonectomia esquerda, o que demonstra que as duas técnicas de sutura são adequadas nos cães.

O modelo experimental desenvolvido demonstra que a técnica de pneumonectomia 
Tabela 1 - Resultados dos exames histopatológicos em todos os cães dos Grupos Ae B aos 7,15 e 36 dias de pós-operatório

\begin{tabular}{|c|c|c|c|c|c|c|c|}
\hline Cães & Cirurgia & $\begin{array}{c}\text { Tecido de } \\
\text { granulação }\end{array}$ & Fibrose & $\begin{array}{l}\text { Granuloma tipo } \\
\text { corpo estranho }\end{array}$ & $\begin{array}{l}\text { Vasos neo } \\
\text { formados }\end{array}$ & $\begin{array}{l}\text { Inflama- } \\
\text { ção }\end{array}$ & $\begin{array}{l}\text { Necro- } \\
\text { se }\end{array}$ \\
\hline 1 & $\begin{array}{c}\text { Sutura Manual } \\
7 \text { dias }\end{array}$ & Sim & + & Não & ++ & ++ & Não \\
\hline 2 & $\begin{array}{c}\text { Sutura Manual } \\
7 \text { dias }\end{array}$ & Sim & + & Sim & +++ & ++ & Sim \\
\hline 3 & $\begin{array}{c}\text { Sutura Manual } \\
7 \text { dias } \\
\end{array}$ & Sim & +++ & Sim & +++ & +++ & Não \\
\hline 4 & $\begin{array}{c}\text { Sutura Manual } \\
15 \text { dias }\end{array}$ & Sim & +++ & Sim & +++ & +++ & Não \\
\hline 5 & $\begin{array}{c}\text { Sutura Manual } \\
15 \text { dias }\end{array}$ & Sim & +++ & Sim & +++ & +++ & Não \\
\hline 6 & $\begin{array}{c}\text { Sutura Manual } \\
15 \text { dias }\end{array}$ & Sim & +++ & Sim & +++ & +++ & Sim \\
\hline 7 & $\begin{array}{c}\text { Sutura Manual } \\
36 \text { dias }\end{array}$ & Não & +++ & Sim & +++ & +++ & Não \\
\hline 8 & $\begin{array}{c}\text { Sutura Manual } \\
36 \text { dias }\end{array}$ & Não & +++ & Sim & +++ & +++ & Sim \\
\hline 9 & $\begin{array}{c}\text { Sutura Manual } \\
36 \text { dias }\end{array}$ & Não & +++ & Sim & ++ & +++ & Não \\
\hline 10 & $\begin{array}{c}\text { Sutura Mecânica } \\
7 \text { dias } \\
\end{array}$ & Sim & +++ & Não & ++ & +++ & Sim \\
\hline 11 & $\begin{array}{c}\text { Sutura Mecânica } \\
7 \text { dias }\end{array}$ & Sim & ++ & Não & ++ & +++ & Sim \\
\hline 12 & $\begin{array}{c}\text { Sutura Mecânica } \\
7 \text { dias } \\
\end{array}$ & Sim & ++ & Não & +++ & + & Não \\
\hline 13 & $\begin{array}{c}\text { Sutura Mecânica } \\
15 \text { dias }\end{array}$ & Sim & +++ & Não & +++ & ++ & Sim \\
\hline 14 & $\begin{array}{c}\text { Sutura Mecânica } \\
15 \text { dias }\end{array}$ & Sim & +++ & Não & +++ & + & Não \\
\hline 15 & $\begin{array}{c}\text { Sutura Mecânica } \\
15 \text { dias } \\
\end{array}$ & Sim & ++ & Não & ++ & ++ & Não \\
\hline 16 & $\begin{array}{c}\text { Sutura Mecânica } \\
36 \text { dias }\end{array}$ & Não & +++ & Não & + & + & Sim \\
\hline 17 & $\begin{array}{c}\text { Sutura Mecânica } \\
36 \text { dias } \\
\end{array}$ & Não & +++ & Não & + & + & Sim \\
\hline 18 & $\begin{array}{c}\text { Sutura Mecânica } \\
36 \text { dias }\end{array}$ & Não & +++ & Não & + & + & Não \\
\hline
\end{tabular}

+ : leve; + +: moderado; +++ : intenso

esquerda é plenamente viável no cão, permitindo o estudo comparativo entre sutura manual e mecânica. Todos os cães sobreviveram ao período pós-operatório não apresentando complicações quanto à ferida operatória.

Não encontramos na literatura dados pertinentes à avaliação histopatológica de cirurgias experimentais, comparando sutura manual e mecânica em brônquio principal após pneumonectomia esquerda em cães. Inúmeros trabalhos comparando sutura manual e mecânica após pneumonectomia foram realizados. No entanto, por se tratarem de pacientes humanos, os autores não realizaram avaliação anatomopatológica.

Encontramos poucas pesquisas sobre avaliação histopatológica, comparando sutura manual e mecânica em brônquio após lobectomia experimental em cães. Estes trabalhos foram realizados por PêgoFernandes et al. ${ }^{13}$ e Ferreira et al. ${ }^{14}$.

No presente trabalho, a sutura manual causou uma reação inflamatória tecidual mais intensa, independente do tempo de sutura. Este predomínio ficou evidente aos 7, 15 e 36 dias de pós-operatório. A duração e a intensidade da reação inflamatória variam com o tipo de material usado para a realização da sutura, técnica cirúrgica, tecido em que a sutura é feita, além de outros fatores.

Concordamos com Wood, Collins e Walshaw ${ }^{28}$ quando citam que os grampos de titânio, quimicamente inertes, causam reação inflamatória mínima, já que no presente estudo, a histopatologia revelou menor inflamação e ausência de granuloma tipo 
corpo estranho nos cães submetidos à sutura mecânica. Por outro lado, todos os cães submetidos à sutura manual, com exceção do cão $\mathrm{n}^{\circ} 1$, apresentaram granuloma tipo corpo estranho.

Constatamos que em relação ao tecido de granulação, fibrose, neoformação vascular e necrose não houve diferença entre sutura manual e mecânica.

As características histológicas semelhantes, estudadas após a realização de um estudo comparativo entre sutura manual e mecânica em brônquio após lobectomia experimental em cães, também são referidas por Pêgo-Fernandes et al. ${ }^{13}$.

Resultados diferentes foram apresentados por Ferreira et al. ${ }^{14}$. Realizaram um estudo comparativo entre sutura manual com fio de poliéster e sutura mecânica após bilobectomia. Com relação ao processo cicatricial, não foram encontradas diferenças na avaliação histopatológica.

No presente estudo, o exame macroscópico da cavidade torácica, realizado durante a necrópsia nos cães de ambos os Grupos, demonstrou aderências na região do hilo pulmonar de pouca significância. Não se notaram sinais de infecção cirúrgica e deiscência junto às suturas da artéria pulmonar, veias pulmonares, brônquio principal esquerdo e musculatura intercostal.

Portanto, os dois tipos de sutura proporcionaram uma cicatrização adequada em todos os tempos de observação.

\section{Conclusões}

Embora tenha havido maior intensidade de inflamação e maior ocorrência de granuloma tipo corpo estranho nos cães submetidos à sutura manual, o resultado após cicatrização foi semelhante nos 2 grupos. Portanto, podemos afirmar que os 2 tipos de sutura promoveram cicatrização segura e eficiente do coto brônquico principal esquerdo, sem a presença de fístula brônquica nos cães dos Grupos A e B.

\section{Agradecimentos}

Trabalho conduzido com o apoio financeiro da Fundação de Amparo à Pesquisa do Estado de São Paulo (FAPESP)

\section{Comparative study between mechanical and manual sutures in the bronchus after left pneumonectomy in the dogs (canis familiaris): a pathological-anatomic evaluation}

\begin{abstract}
The objective of this study was to perform an experimental study to be compared under the pathological-anatomic, point of view, the manual and mechanical sutures in the main bronchus after left pneumonectomy in dogs. Eighteen adult mongrel, healthy dogs, both male and female, were utilized weighing from 9 to $27.5 \mathrm{~kg}$. The dogs were submitted to a selective intubation and left thorax incision in the 5 th intercostal space where a pneumonectomy was performed. Were separated into 2 groups of 9 dogs according to the type of suture employed: Group A - a manual suture with polypropylene 5-O; Group B - a mechanical suture with a mechanical stapler, model TL-30. Each group was subdivided into 3 subgroups of 3 animals and a temporal postoperative study was established at 7, 15 and 36 days where an pathological-anatomic evaluation was made on the healing of the manual and mechanical sutures. During the histopathological evaluation, the intensity of inflammation, fibrosis, neoformed vessels, and the presence or absence of granulation tissue, foreign body reaction and necrosis
\end{abstract}

Key-words:

Suture.

Bronchus.

Pneumonectomy.

Dogs 
were evaluated qualitatively and semi-quantitatively. The results found were analyzed statistically. Regarding the histopathologic analysis, foreign body reaction occured in the left bronchial stump in $88,9 \%$ of the dogs submitted to a manual suture and in none of the dogs submitted to a mechanical suture. There is still, significant statistical difference in the dogs in Groups A and B in relation to the intensity of the inflammation, the greatest intensity being in the dogs submitted to the manual suture. It is concluded that both types of sutures brought an adequate healing of the main left bronchial stump, although there was a greater intensity of inflammation and a greater occurence of foreign body reaction in the dogs submitted to the manual suture.

\section{Referências}

1 DENANS, F. N. Lettre avec envoi $d^{\prime}$ un systene de viroles qu' il propose en remplacement de la ligature pour la réunion de plaies transversales de $I^{\prime}$ intestin. Bulletin de I' Académe Royale de Médecine, p. 719, 1836.

2 SCHWARTZ, A. Historical and veterinary perspectives of surgical stapling. The Veterinary Clinics of North America: Small Animal Practice, v. 24, n. 2, p. 225-246, 1994.

3 STEICHEN, F. M.; RAVITCH, M. M. Mechanical sutures in surgery. British Journal of Surgery, v. 60, n. 3 , p. 191-197, 1973.

4 PETERFFY, A.; CALABRESE, E. Mechanical and conventional manual sutures of the bronchial stump - a comparative study of 298 patients. Scand Journal of Thoracic Cardiovascular Surgery, v. 13, p. 87-91, 1979.

5 LARUE, S. M.; WITHROW, S. J.; WYKES, P. M. Lung resection using surgical staplers in dogs and cats. Veterinary Surgery, v. 16, n. 3, p. 238-240, 1987.

6 HOOD, R. M. Stapling techniques involving lung parenchyma. Surgery Clinic North America, v. 64, n. 3, p. 469-480, 1984.

7 JATENE, F. B. et al. Uso de grampeadores mecânicos em ressecção broncopulmonar. Jornal de Pneumologia, v. 22, n. 6, p. 291-294, 1996.

8 TAKARO, T. Use of staplers in pulmonary surgery. Surgery Clinic of North America, v. 64, n. 3, p. 461468, 1984.

9 WALSHAW, R. Stapling techniques in pulmonary surgery. The Veterinary Clinics of North America. Small Animal Practice, v. 24, n. 2, p. 335-366, 1994.

10 AMOSOV, N. M.; BEREZOVSKI, K. K. Pulmonary resection with mechanical suture. The Journal of Thoracic and Cardiovascular Surgery, v. 41, p. 325-335, 1961.

11 DART, C. H.; SCOTT, S. M.; TAKARO, T. Six years clinical experience using automatic stapling devices for lung resections. Annals of Thoracic Surgery, v. 9, n. 6, p. 535-550, 1970.

12 FORRESTER-WOOD, C. P. Bronchopleural fistula following pneumonectomy for carcinoma of the bronchus. The Journal of Thoracic and Cardiovascular Surgery, v. 80, n. 3, p. 406-409, 1980.

13 PÊGO-FERNANDES, P. et al. Estudo comparativo entre sutura mecânica e manual em brônquios de cães. Jornal de Pneumologia, v. 2, n. 16, p. 71-74, 1990.

14 FERREIRA, J. A. D. et al. Comparação entre sutura convencional com fio de poliéster e sutura com grampos de aço inoxidável na lobectomia parcial pulmonar. Estudo experimental em cães (Canis familiaris). Revista Brasileira de Ciência Veterinária, v. 4, n. 3, p. 127-130, 1997.

15 WEISSBERG, D.; KAUFMAN, M. Suture closure versus stapling of bronchial stumps in 304 lung cancer operations. Scand Journal of Thoracic Cardiovascular Surgery, v. 26, n. 2, p. 125-127, 1992.

16 HUBAUT, J. J. et al. Closure of the bronchial stump by manual suture and incidence of bronchopleural fistula in a series of 209 pneumonectomies for lung cancer. European Journal of Cardio-Thoracic Surgery, v. 16 , n. 4, p. 418-423, 1999.

17 ASAMURA, H.; KONDO, H.; TSUCHIYA, R. Management of the bronchial stump in pulmonary resections: a review of 533 consecutive recent bronchial closures. European Journal of Cardio-Thoracic Surgery, v. 17 , p. $106-110,2000$

18 ASAMURA, $\mathrm{H}$. et al. Bronchopleural fistulas associated with lung cancer operations. Univariates and multivariates analysis of risk factors, management and outcome. The Journal of Thoracic and Cardiovascular Surgery, n. 104, p. 1456-1464, 1992.

19 ENG, J.; SABANATHAN, S. Tissue adhesive in bronchial closure. Annals of Thoracic Surgery, v. 48, p. 683-685, 1989.

20 HOLLAUS, P. H. et al. Natural history of bronchopleural fistula after pneumonectomy: a review 
of 96 cases. Annals of Thoracic Surgery, n. 63, p. 13911397, 1997.

21 HOLLAUS, P. H. et al. Endoscopic treatment of postoperative bronchopleural fistula: experience with 45 cases. Annals of Thoracic Surgery, v. 66, p. 923927, 1998.

22 RIVIERE, A. B. et al. Transternal closure of bronchopleural fistula after pneumonectomy. Annals of Thoracic Surgery, v. 64, p. 954-959, 1997.

23 SAWASAKI, H. et al. Postoperative bronchopleural fistula: clinical and experimental study. Chest, v. 67, n. 6, p. 702-705, 1975

24 WATANABE, S.; WATANABE, T.; URAYAMA, H. Endobronchial occlusion method of bronchopleural fistula with metallic coils and glue. Thorac Cardiovascular Surgery, v. 51, n. 2, p. 106-108, 2003.

25 WEBER, J. et al. Empyema after pneumonectomy empyema window or thoracoplasty? Thorac Cardiovascular Surgeon, v. 38, n. 6, p. 355-358, 1990.

26 WEISSBERG, D.; KAUFMAN, M. Suture closure versus stapling of bronchial stumps in 304 lung cancer operations. Scand Journal of Thoracic Cardiovascular Surgery, v. 26, n. 2, p. 125-127, 1992.

27 WRIGHT, C. D. et al. Postpneumonectomy bronchopleural fistula after sutured bronchial closure: incidence, risk factors, and management. The Journal of Thoracic and Cardiovascular Surgery, v. 112, n. 5, p. 1367-1371, 1996

28 WOOD, D. S.; COLLINS, J. E.; WALSHAW, R. Tissue reaction to nonabsorbable suture materials in the canine linea alba: a histological evaluation. Journal of the American Animal Hospital Association, v. 20, p. 39-44, 1984. 